\title{
The microbiome of the leaf surface of Arabidopsis protects against a fungal pathogen
}

\author{
Unyarat Ritpitakphong ${ }^{1}$, Laurent Falquet ${ }^{1,2}$, Artit Vimoltust ${ }^{3}$, Antoine Berger ${ }^{1}$, Jean-Pierre Métraux ${ }^{1}$ and $^{2}$ \\ Floriane L'Haridon ${ }^{1}$ \\ ${ }^{1}$ Department of Biology, University of Fribourg, 10 chemin du Musée, CH-1700 Fribourg, Switzerland; ${ }^{2}$ Swiss Institute of Bioinformatics, University of Fribourg, 10 Chemin du Musée, \\ CH-1700 Fribourg, Switzerland; ${ }^{3}$ Product \& Technology Development Center, SCG Paper Plc, 1 Siam Cement Road, Bangsue, Bangkok 10800, Thailand
}

\author{
Author for correspondence: \\ Floriane L'Haridon \\ Tel: +41263008811 \\ Email: floriane.Iharidon@unifr.ch \\ Received: 1 October 2015 \\ Accepted: 13 November 2015
}

New Phytologist (2016)

doi: $10.1111 / \mathrm{nph} .13808$

Key words: Arabidopsis thaliana, Botrytis cinerea, cuticle permeability, phyllosphere diversity, plant protection, Pseudomonas.

\section{Summary}

- We have explored the importance of the phyllosphere microbiome in plant resistance in the cuticle mutants $b d g$ (BODYGUARD) or lacs2.3 (LONG CHAIN FATTY ACID SYNTHASE 2) that are strongly resistant to the fungal pathogen Botrytis cinerea.

- The study includes infection of plants under sterile conditions, 165 ribosomal DNA sequencing of the phyllosphere microbiome, and isolation and high coverage sequencing of bacteria from the phyllosphere.

- When inoculated under sterile conditions bdg became as susceptible as wild-type (WT) plants whereas lacs2.3 mutants retained the resistance. Adding washes of its phyllosphere microbiome could restore the resistance of $b d g$ mutants, whereas the resistance of lacs 2.3 results from endogenous mechanisms. The phyllosphere microbiome showed distinct populations in WT plants compared to cuticle mutants. One species identified as Pseudomonas sp isolated from the microbiome of $b d g$ provided resistance to B. cinerea on Arabidopsis thaliana as well as on apple fruits. No direct activity was observed against $B$. cinerea and the action of the bacterium required the plant.

- Thus, microbes present on the plant surface contribute to the resistance to $B$. cinerea. These results open new perspectives on the function of the leaf microbiome in the protection of plants.

\section{Introduction}

The nonpathogenic microbiota, often referred to as neutral microbes, turn out to contribute in various ways to the development and to the health of the individuals harbouring them. For example, the microbiome in the human gut has been linked with a healthy function of the brain, the immune system, the digestive system, and with a number of diseases ranging from cancer to metabolic or even psychiatric disorders (Foster \& McVey Neufeld, 2013; Biedermann \& Rogler, 2015; Dash et al., 2015; Viaud et al., 2015). In animals, the microbiome has been associated with similar functions in development and disease (Engel \& Moran, 2013; Kostic et al., 2013; Sabree \& Moran, 2014). A lot of interest has also been given to microbes associated with surfaces of roots or leaves (Andrews \& Harris, 2000; Lindow \& Brandl, 2003; Vorholt, 2012; Humphrey etal., 2014). Such microbes are proposed to be associated with protection and nutrient acquisition (Bulgarelli et al., 2013). Soil types and associated abiotic and biotic parameters, as well as chemicals secreted by roots, create a niche for specific microbes and determine plantspecific rhizosphere communities that help with protection and nutrient acquisition (Walker et al., 2003; Bulgarelli et al., 2013).
Bacteria predominantly colonize the phyllosphere (Lindow \& Brandl, 2003) where environmental conditions together with surface features determine the composition of the phyllosphere flora (Vorholt, 2012). For instance, single mutations in genes controlling wax biosynthesis in Arabidopsis thaliana lead to distinct chemical composition of waxes together with distinct bacterial communities on the phylloplane (Reisberg et al., 2013). The phylloplane of $A$. thaliana plants grown under similar sterile conditions and inoculated initially with a same representative mixture of the most abundant phylloplane bacteria was analysed in a collection of mutants with phenotypes ranging from altered cell walls to impaired defences and secondary metabolism. Interestingly, the strongest difference in bacterial composition and abundance compared to the wild-type plants was observed in lacs2.3, lacs 2.4 and pec1 mutants affected in the biosynthesis of the cuticle (Bodenhausen et al., 2014). Mutants affected in the structure of the cuticle are characterized by enhanced cuticular permeability and display enhanced resistance to the agriculturally relevant fungal pathogen Botrytis cinerea. In addition to resistance, many of these cuticular mutants spontaneously accumulate reactive oxygen species (Serrano et al., 2014). In this study we have tested the hypothesis that the phyllosphere microbes including epiphytes 
and endophytes contribute to the resistance of $A$. thaliana to $B$. cinerea with a special emphasis on the cuticle.

\section{Materials and Methods}

\section{Plant maintenance}

The nonsterile Arabidopsis thaliana (L.) Heynh seeds were grown on a pasteurized soil mix of humus and perlite $(3: 1)$. Seeds were kept at $4^{\circ} \mathrm{C}$ for $2 \mathrm{~d}$ and then transferred to the growth chamber. Plants were grown in a $12 \mathrm{~h}: 12 \mathrm{~h}$, light : dark cycle with $65 \%$ of relative humidity, with a day temperature of $22^{\circ} \mathrm{C}$ and a night temperature of $19^{\circ} \mathrm{C}$. Wild-type (WT) Col-0 plants were obtained from the Nottingham Arabidopsis Stock Center (Nottingham, UK). The mutant referred to as $b d g$ is bdg2 (Kurdyukov etal., 2006) and lacs2.3 is described in (Bessire etal., 2007). The fls2 (SALK_062054C) mutant and the mutant referred to as efr was efr-1 (SALK_044334), both previously described by Zipfel et al. (2004, 2006). The A.thaliana mutant referred to as sid2 was sid2-1, etr1 was etr1-1, ein2 was ein2-1 and jar1 was jar1-1, as described in Chassot et al. (2007). Arabidopsis mutant dde2-2 was previously described (von Malek et al., 2002).

For sterilization, seeds were placed in a tube and shaken in $70 \%$ ethanol for $35 \mathrm{~min}$ at room temperature. After centrifugation at full speed, the ethanol was removed and absolute ethanol was added to the tube and immediately centrifuged at full speed. The ethanol was then removed and the seeds were placed in a sterile cabinet to dry. The sterile $A$. thaliana seeds of Col-0, $b d g$ and lacs 2.3 were grown on $0.5 \times \mathrm{MS}$ agar plates $\left(2.16 \mathrm{gl}^{-1}\right.$ Murashige and Skoog basal salt mixture, $0.1 \mathrm{~g} \mathrm{l}^{-1}$ Murashige and Skoog vitamin, $10 \mathrm{~g}^{-1}$ sucrose, $\mathrm{pH} 5.7(\mathrm{KOH}), 8 \mathrm{gl}^{-1}$ bacteriological agar). The seeds were transferred to a growth chamber for $7 \mathrm{~d}$ in a $16 \mathrm{~h}: 8 \mathrm{~h}$, light : dark cycle with $45 \%$ humidity and temperatures of $22^{\circ} \mathrm{C}$ (day) and $19^{\circ} \mathrm{C}$ (night). The seedlings were transferred to a sterile box (Model TP1200+ TPD1200, filter code XXL; www.microbox-container.com) and grown on MS agar medium (4.33 $\mathrm{g} \mathrm{l}^{-1}$ Murashige and Skoog basal salt mixture, $0.1 \mathrm{~g}^{-1}$ Murashige and Skoog vitamin, $30 \mathrm{~g} \mathrm{l}^{-1}$ sucrose, $\mathrm{pH} 5.7$ $(\mathrm{KOH}), 7 \mathrm{~g}^{-1}$ bacteriological agar) in the growth chamber under the same conditions as those used for the nonsterile seeds. The sterility of the plant material was assessed by growing plant homogenates on Petri dishes for several weeks.

\section{Culture of $B$. cinerea, inoculation and staining of hyphae}

Botrytis cinerea strains BMM, provided by Brigitte Mauch-Mani (University of Neuchâtel, Switzerland), were grown on Difco (Becton Dickinson; http://www.bd.com) $39 \mathrm{~g} \mathrm{l}^{-1}$ potato dextrose agar. Spores were harvested in water and filtered through glass wool to remove hyphae. Spores were diluted in $0.25 \times$ Difco potato dextrose broth (PDB 1/4; $6 \mathrm{gl}^{-1}$ ) at $5 \times 10^{4}$ spores $\mathrm{ml}^{-1}$ for inoculation. Droplets of $6 \mu \mathrm{l}$ spore suspension at $5 \times 10^{4}$ spores $\mathrm{ml}^{-1}$ were deposited on nonsterile leaves of 4-wk-old plants and on sterile leaves of 5-wk-old plants. The nonsterile plants were then incubated in the growth chamber under high humidity in covered trays with water in the dark. The sterile plants were kept under high humidity by adding $2 \mathrm{ml}$ of sterile water in a sterile box. The quantification of lesion size (in $\mathrm{mm}$ ) was performed after $3 \mathrm{~d}$ of infection.

The treatment with microbes of phyllosphere and the infection with $B$. cinerea of nonsterile plants were performed by mixing B. cinerea at $5 \times 10^{4}$ spores $\mathrm{ml}^{-1}$ to the phyllosphere microbes resuspended in PDB 1/4. This protocol was also used for inoculation of $B$. cinerea and strains 1,2 and 3 on both sterile and nonsterile plants. The infection of sterile plants treated with microbes from leaf surfaces was performed by inoculation of leaves with $6 \mu \mathrm{l}$ of microbe solution. The plants were incubated for $1 \mathrm{~d}$ in the growth chamber then infected with B. cinerea at $5 \times 10^{4}$ spores $\mathrm{ml}^{-1}$. Fungal structures and dead plant cells were stained by boiling inoculated leaves for $5 \mathrm{~min}$ in a solution of alcoholic lactophenol trypan blue. Stained leaves were extensively cleared in chloral hydrate $\left(2.5 \mathrm{~g} \mathrm{ml}^{-1}\right)$ at room temperature by gentle shaking, and then observed using a Leica DMR microscope with bright-field settings.

\section{Sampling of the microbes from the phyllosphere}

For each ecotype, one hundred leaf discs $\left(12.6 \mathrm{~mm}^{2}\right)$ were punched out with a cork borer and $15 \mu \mathrm{l}$ per disc of $0.1 \mathrm{M}$ potassium phosphate buffer $\mathrm{pH} 8$ were added to the tubes. For the treatment of plants grown under nonsterile conditions, the samples were sonicated in a water bath $(47 \mathrm{kHz} \pm 6 \%)$ for 2 min and vortexed for $30 \mathrm{~s}$, this step was repeated two more times. The supernatant was placed in a new tube and centrifuged at $12000 \mathrm{~g}$ for $20 \mathrm{~min}$ at $4^{\circ} \mathrm{C}$. The pellet was resuspended with $12 \mu \mathrm{l}$ of PDB $1 / 4$ per leaf disc. This was considered to be the undiluted bacterial concentration of the phyllosphere. For the treatment of sterile plants with microbes from the phyllosphere, the samples were shaken for $15 \mathrm{~min}$ at $25 \mathrm{~Hz}$ by using a mixer mill MM400 (www.rescht.com) and sonicated in a water bath $(47 \mathrm{kHz} \pm 6 \%$ ) for $5 \mathrm{~min}$. The pellet was resuspended with $6 \mu \mathrm{l}$ of $\mathrm{H}_{2} \mathrm{O}$ per leaf disc.

For sequencing, the entire leaves were weighed and placed in $10 \mathrm{ml}$ of $0.1 \mathrm{M}$ potassium phosphate buffer $\mathrm{pH} 8$ per gram of leaf material. For the extraction of phyllosphere bacteria, the protocol described above for experiments in nonsterile condition was followed.

\section{Bacterial cultures}

Bacterial strains 1, 2 and 3 were spread on Luria Broth (LB) agar medium for $24 \mathrm{~h}$ at $28^{\circ} \mathrm{C}$, one colony obtained for each strain was spread again on a new LB agar plate and incubated for $24 \mathrm{~h}$ at $28^{\circ} \mathrm{C}$. Some colonies were used from this plate and diluted in $100 \mu \mathrm{l}$ of PDB $1 / 4$ and mixed. The optical density (OD) at $600 \mathrm{~nm}$ was measured. The bacterial suspension was diluted to obtain the approximate value presented in the Table 1 . The bacterial suspension was then diluted in a 10-times series dilution until the expected concentration was obtained. For the experiment using boiled bacteria strains, the bacteria were incubated in hot water at $100^{\circ} \mathrm{C}$ for $25 \mathrm{~min}$. 
Table 1 Correspondence between optical density (OD) $(600 \mathrm{~nm})$ and the colony-forming units (CFU) $\mathrm{\mu l}^{-1}$ for three bacterial strains isolated from the phyllosphere

\begin{tabular}{lll}
\hline Species & OD $(600 \mathrm{~nm})$ & $\mathrm{CFU} \mu \mathrm{I}^{-1}$ \\
\hline Strain 1 & 0.027 & $4 \times 10^{4}$ \\
Strain 2 & 0.011 & $1 \times 10^{3}$ \\
Strain 3 & 0.015 & $4 \times 10^{4}$ \\
\hline
\end{tabular}

\section{Apple fruit infection}

Apple fruit were disinfected in 2\% sodium hypochlorite solution for $2 \mathrm{~min}$ and rinsed with sterile tap water. The spore suspension of $B$. cinerea was adjusted at $5 \times 10^{4}$ spores $\mathrm{ml}^{-1}$ in PDB $1 / 4$ or in a suspension of strain 3 . The apples were inoculated with $10 \mu \mathrm{l}$ of suspension at eight sites that were wounded with a sterile needle and one wounded site was inoculated with PDB 1/4. The apples were put in covered trays at very high humidity and incubated $5 \mathrm{~d}$ in the growth chamber without direct light. Two diameters of lesions were measured for each inoculated site according to the protocol described in http://www.bio-protocol.org/e1311.

\section{Estimation of bacterial population size}

The microbes isolated from Col- $0, b d g$, lacs 2.3 or bacterial strains 1, 2 and 3 were estimated by dilution plating and expressed in colony-forming units (CFU). Twenty-five microlitres of bacterial solution were spread on LB agar plates. The plates were incubated for $24 \mathrm{~h}$ at $28^{\circ} \mathrm{C}$ and the number of colonies were determined.

\section{In vitro toxicity of microbes from the phyllosphere on the} growth of $B$. cinerea

Microbes from the phyllosphere $(13.5 \mu \mathrm{l})$ diluted in PDB $1 / 4$ were mixed with $1.5 \mu \mathrm{l}$ of $B$. cinerea spores in PDB $1 / 4$ to a final concentration of $5 \times 10^{4}$ spores $\mathrm{ml}^{-1}$ and placed on a glass slide. The glass slide was placed in a humid box and incubated for $16 \mathrm{~h}$ in a growth chamber. The growth of fungus was then observed under a microscope.

\section{DNA extraction of samples for sequencing}

Entire leaves were collected from 12 different plants per sample and five samples per ecotype were prepared. Twenty millilitres of $0.1 \mathrm{M}$ potassium phosphate buffer $\mathrm{pH} 8$ were added to the tubes. The samples were sonicated in a water bath $(47 \mathrm{kHz} \pm 6 \%)$ for $2 \mathrm{~min}$ and vortexed for $30 \mathrm{~s}$; this step was repeated two more times. The pellet of microbes was obtained after centrifugation at $12000 \mathrm{~g}$ for $20 \mathrm{~min}$ at $4^{\circ} \mathrm{C}$. The pellet of microbes was resuspended with the solution present in the PowerBead tubes; DNA of microbes was extracted using the Power Soil DNA Kit (http://www.mobio.com). The standard protocol described was followed but the volume of elution was $60 \mu \mathrm{l}$ of water. Three independent experiments were performed. DNA of $250 \mathrm{mg} \mathrm{sam}-$ ples of soil where Col-0, bdg and lacs2.3 had grown (respectively) was extracted following the same procedure. A library was prepared using the primers 799F 5'-AACMGGATTAGAT ACCCKG-3' and 1193R 5'-ACGTCATCCCCACCTTCC-3', as previously described (Bodenhausen et al., 2013). The samples were then sent to Microsynth (http://www.microsynth.ch) for sequencing using the Illumina kit (http://www.illumina.com).

\section{Microbial community analysis}

The software package MoTHur (v.1.34.4) was used for sequence analysis (Schloss et al., 2009), following the Standard Operating Procedure outlined on http://www.mothur.org/wiki/MiSeq _SOP. Briefly, the two overlapping paired-end reads were combined using make.contig. Then, each unique sequence was aligned with align.seqs to the SILVA reference alignment release 119 (Quast et al., 2013). A distance matrix was calculated allowing for four mismatches. Chimeric sequences were identified using chimera.uchime and removed. Sequences matching 'Chloroplast-Mitochondria-unknown-Archaea-Eukaryota' were also removed. Next, sequences were clustered using the furthest neighbour clustering algorithm to build OTUs (operational taxonomic unit). The resulting file was parsed to separate the data for each sample. OTUs were assigned a taxonomic group with classify.seqs using the RDP reference file (Wang et al., 2007) and a cut-off of $80 \%$ of the bootstrap value. For the description of the community, the sequences are split at the order level and OTUs with the same taxonomy were clustered together at a cut-off level of 0.03. Metagenassist (Arndt et al., 2012) was used to calculate several statistics on the output of MOTHUR. Briefly, data were filtered by interquartile range (IQR) and normalized row- and column-wise by sum and Pareto scaling. The dendrogram and heat map were calculated at genus level with Spearman distance and Ward clustering algorithm. MEGAN (Huson et al., 2011) was used to calculate the rarefaction curves after converting data to the biom format with MoTHur.

\section{DNA bacteria extraction for Pacific Bioscience sequencing}

Bacterial strains 1 and 3 were spread on LB agar medium for $24 \mathrm{~h}$ at $28^{\circ} \mathrm{C}$. One colony was resuspended in $3 \mathrm{ml}$ of $\mathrm{LB}$ liquid and shaken at $200 \mathrm{~min}^{-1}$ at $28^{\circ} \mathrm{C}$ during $24 \mathrm{~h}$ in an incubator. The DNA was extracted by using a GenElute ${ }^{\mathrm{TM}}$ bacterial genomic DNA kit (Sigma-Aldrich). The protocol for Gram-negative DNA extraction described was followed, but the wash step was repeated three times and the elution volume was $100 \mu \mathrm{l}$ of water. The DNA samples were sent to the Center for Integrative Genomic at the University of Lausanne. The libraries for Pacific Bioscience sequencing were prepared as recommended by the manufacturer, and sequenced on two SMRT cells (one for each library), leading to $800-950 \mathrm{Mbp}$ of data. The reads of each SMRT cell were assembled using the SMRT pipeline (v.2.3) with the protocol HGAP3 (Chin et al., 2013) leading to a single contig for genome 3, of $6374437 \mathrm{bp}$. The GC content of genome 3 is $59.23 \%$. Similarly, genome 1 led to a single contig of $4835345 \mathrm{bp}$ and a GC content of $61.7 \%$. No plasmid was detected for both genomes 1 and 3 . 


\section{Annotation, accession numbers and genome comparison}

The genomes of strain 1 and strain 3 were annotated using PROKKA (Seemann, 2014) and deposited in the ENA database (Silvester et al., 2014) under project PRJEB9391 (accession numbers: LN865164 for strain 1 and LN854573 for strain 3; ERR906813-ERR906860 for the 16S rRNA raw reads runs). The genomes of strain 1 and strain 3 were compared with MAUVE (Darling et al., 2010) and BRIG (Alikhan et al., 2011).

\section{DNA extraction and qPCR for bacteria quantification}

Leaves of 12 different plants from Col0, bdg and lacs 2.3 were collected and frozen in liquid nitrogen. The leaves were ground and DNA was extracted from $100 \mathrm{mg}$ of powder by using peqGold Plant DNA mini kit (http://www.peglab.com) following the protocol described, $40 \mu \mathrm{l}$ of elution water was used. DNA was quantified using nanodrop (http://www.nanodrop.com). Ten microlitres of DNA at $10 \mathrm{ng} \mu \mathrm{l}^{-1}$ were mixed with $12.5 \mu \mathrm{l}$ of Sensimix SYBR Green kit (http://www.bioline.com), $0.75 \mu \mathrm{l}$ of primers at $10 \mu \mathrm{M}$ and $1 \mu \mathrm{l}$ of $\mathrm{H}_{2} \mathrm{O}$. The specific primers nicA for $5^{\prime}$-GGATCCCT CCATCATCTGG-3' and nicA Rev 5'-CCCAGACCTTCTAC GGTACG-3' were used to amplify the nicotinate dehydrogenase subunit A (nicA) gene of bacteria strain 3. The gene expression of nicA was normalized to the expression of the reference plant gene AT5g26751, a SHAGGY-related kinase gene (AtSK). The primers used for the reference gene of $A$. thaliana was iASK1 5'-CTTAT CGGATTTCTCTATGTTTGGC-3' and iASK2 5'-GAGCTCC TGTTTATTTAACTTGTACATACC- $3^{\prime}$. This reference gene was previously described and used to quantify $B$. cinerea in leaves (Gachon \& Saindrenan, 2004). The qPCR was performed using the program described in the publication of (Gachon \& Saindrenan, 2004).

\section{Statistical analyses}

One-way ANOVA followed by Tukey's test was performed using the SPSS statistics program (University of Fribourg license; IBM company). For comparison between only two samples, a $t$-test was used. Different letters or asterisks above each bar represent statistically significant differences (Tukey's test and $t$-test; $P<0.05)$. The difference in abundance of bacterial genera was tested pairwise between $b d g$ and the other samples (lacs2.3, Col-0, soil) using the Metastats website (White et al., 2009). Metastats is based on the metagenomeSeq $\mathrm{R}$ package and allows comparison of metagenomic samples on the basis of count data. We used 100 permutations of the data ( $b d g n=15$, Col- $0 n=15$, lacs2.3 $n=15$, soil $n=3$ ) to estimate the null distribution. $P$-values below 0.05 were considered significant.

\section{Results}

The importance of the leaf phyllosphere in the resistance to pathogens

In order to determine the implication of phyllosphere microbes in the resistance of $A$. thaliana to pathogens, seeds of WT Col-0 and cuticular mutants $b d g$ and lacs2.3 were surface-sterilized, grown under sterile conditions and inoculated with $B$. cinerea. Under sterile conditions, the $b d g$ mutant lost a large part of its resistance to B. cinerea compared to nonsterile conditions. By contrast, the lacs 2.3 mutant was equally resistant and WT was susceptible in both conditions (Fig. 1). Botrytis cinerea displayed clear hyphal growth as visualized by Trypan blue staining in Col0 leaves in sterile and nonsterile conditions, as well as in leaves of $b d g$ under sterile conditions (Supporting Information Fig. S1). Thus, the microbes of the phyllosphere might potentially affect the outcome of an inoculation with the fungal pathogen B. cinerea.

In order to further test their importance, the phyllosphere microbes were washed off leaves of unsterile plants and reintroduced on leaf surfaces of sterile plants. When $B$. cinerea was inoculated on sterile plants at sites that were pre-treated with the phyllosphere microbes of the corresponding nonsterile plants, $b d g$ became significantly more resistant, whereas lacs 2.3 remained resistant and WT plants remained susceptible to B. cinerea (Fig. 2). In this experiment, bacteria were extracted from a known total surface area and re-applied to a similar area on the receiver plants. This allowed treatment with a bacterial concentration that remained at best equal but more likely slightly lower than that on donor leaves, given unavoidable losses during extraction. This experiment demonstrates the functional importance of phyllosphere microbes in the resistance of $b d g$ to $B$. cinerea.

\section{Is the composition of phyllosphere different in WT and cuticular mutants?}

We tested the hypothesis that cuticular mutants harbour different populations of bacteria on the leaf surface. To this end, we used surface washes of leaf material from plants or samples from the soil mixture where the plants were grown. We analysed the bacterial community by sequencing of the variable regions V5, V6 and V7 of the bacterial $16 \mathrm{~S}$ ribosomal DNA (rDNA) genes. After sequencing we obtained 8191004 raw paired-end reads spread over 48 samples (min 107000 , max 273000 , median 158000

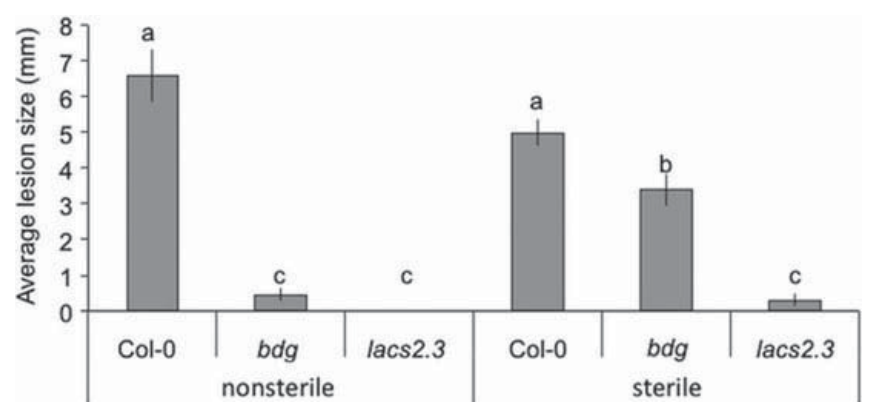

Fig. 1 Resistance of Arabidopsis thaliana Col-0 plants and cuticle mutants against Botrytis cinerea under sterile conditions. Col-0 plants and cuticle mutants ( $b d g$, lacs2.3) were inoculated with $B$. cinerea under nonsterile (left) or sterile conditions (right); $(n=30 ; \pm \mathrm{SE}$ ). The average lesion size was determined $3 \mathrm{~d}$ after inoculation with $B$. cinerea. The experiment was repeated three times with similar results. Different letters above each bar represent statistically significant differences (Tukey's test; $P<0.05$ ). 


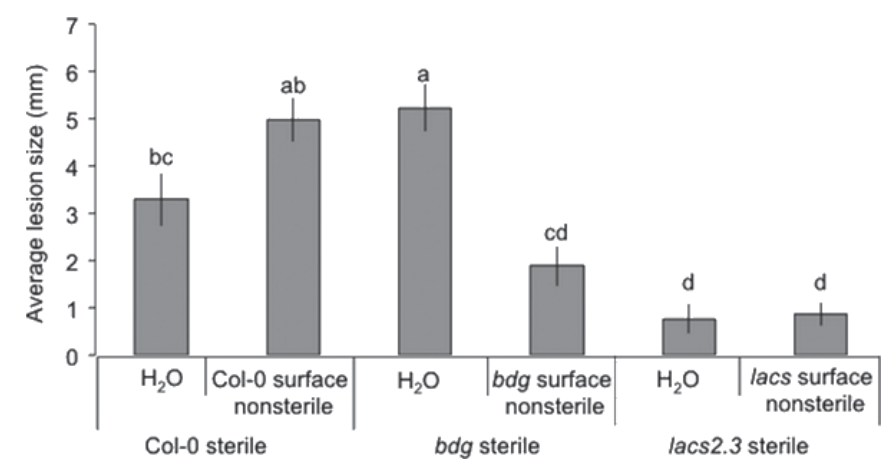

Fig. 2 Resistance of sterile Arabidopsis thaliana Col-0 and cuticle mutants against Botrytis cinerea after treatment with the microbes of their respective phyllosphere extracted from nonsterile plants. Sterile Col-0, bdg and lacs2.3 were pre-treated for $1 \mathrm{~d}$ with their respective phyllosphere microbes from nonsterile plants before infection with $B$. cinerea $(n=30$; $\pm \mathrm{SE}$ ). The average lesion size was determined $3 \mathrm{~d}$ after inoculation with $B$. cinerea. Different letters above each bar represent statistically significant differences (Tukey's test; $P<0.05$ ). The experiment was repeated twice with similar results.

reads). Despite the drop in quality of the MiSeq reads after 200 bp, we obtained 5139594 high quality 413-bp contigs by combining the overlapping forward and reverse reads. Those contigs were further cleaned and analysed with Mothur. The final distribution of $16 \mathrm{~S}$ rRNA unique contigs for the 48 samples was median $66131 \mathrm{~min} 5648$ and $\max 124272$. As shown in the rarefaction curves (Fig. S2), most of the samples (41/48) reached saturation with at least 30000 contigs, meaning that at least $90 \%$ of the diversity was sampled.

The top eight most abundant bacterial genera present in $b d g$ are shown in Fig. 3. As a comparison, the same genera were determined in lacs2.3 and WT plants, as well as in the potting soil (an extension to the 20 most abundant genera in $b d g$ is shown in Fig. S3). There was a clear difference between composition of the genera in cuticle mutants where the most abundant genera were Pseudomonas and Rhizobium. In WT plants and in the soil, Burkholderia was the most abundant genus (Fig. 3). A dendrogram and heat map analysis was used to compare the clustering of the genera in all of the samples collected from the potting soils and surface washes from WT, bdg and lacs2.3 (Fig. 4). It should be noted that the abundances shown in the heat map cannot be directly compared with the mean abundances in Fig. 3 because they represent individual abundances. As expected, the samples from the soils used for WT, bdg and lacs 2.3 all clustered closely together. The samples of washed surfaces of all plants showed a different clustering than that observed in the soil samples indicating that a selection of bacteria had taken place. Furthermore, the bacteria from the leaf washes of WT clustered together and were closer to the soil samples, but could readily be separated from the samples of the leaf washes of the cuticle mutants. By contrast, the bacterial genera from the $b d g$ mutants could not be clearly demarked from those of the lacs 2.3 mutants. This analysis demonstrates that the bacterial community is different on the cuticle mutants compared to WT Col-0 plants. It also highlights the impact of a mutation in a single gene responsible for the cuticular structure on the microbiome of the phyllosphere.

\section{Can the microbes present on the leaf surfaces of cuticular mutants also provide resistance on WT plants?}

Fig. 5 shows that microbes extracted from the phyllospheres of both cuticle mutants were most efficient in inducing resistance in Col- 0 against an infection by $B$. cinerea. The concentration of the bacteria applied to the surface was estimated to be in the same order of magnitude or slightly smaller than that of the donor leaf, assuming that the extraction is complete and the dispersal of the bacteria is uniform on the leaf surface. Microbes from surface washes of WT plants were effective to a lesser extent (Fig. 5). At
Fig. 3 Top eight most abundant genera in $b d g$ and the relative abundance in Arabidopsis thaliana lacs 2.3 mutants, Col-0 plants and soil samples compared to $b d g$ (chosen arbitrarily as a reference because it was the most interesting mutant). Asterisks denote significant difference compared to $b d g(n=15$; error bars denote variance within each respective group; $P<0.05$ ).

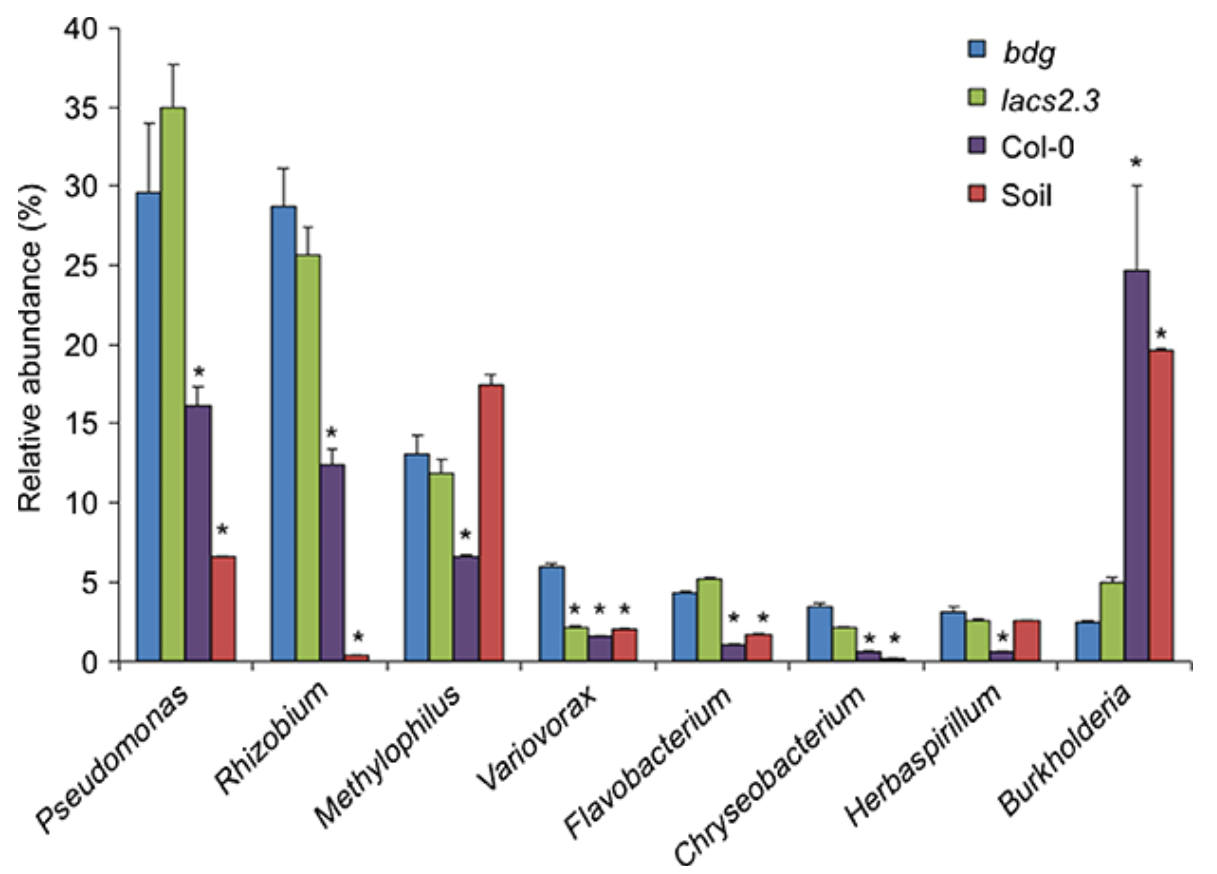




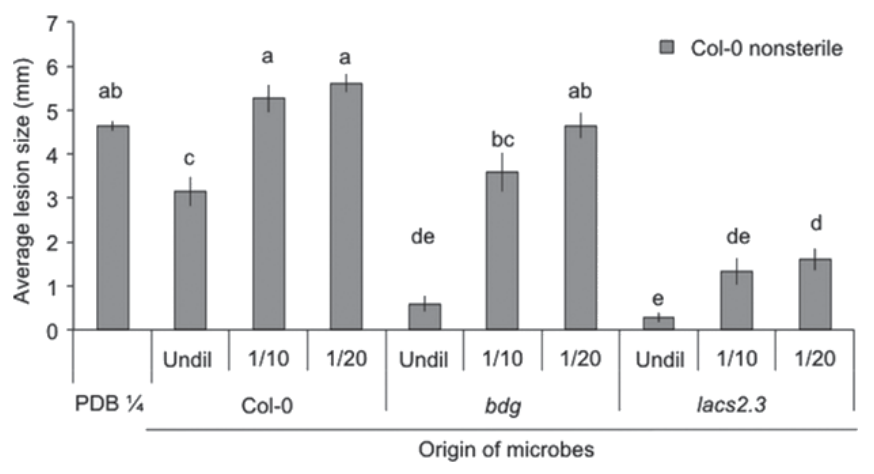

Fig. 5 Effect of microbes extracted from the phyllosphere of Arabidopsis thaliana Col-0 plants and cuticle mutants on the resistance of $A$. thaliana Col-0 plants to Botrytis cinerea. The microbes were washed off leaf surfaces from Col-0, bdg and lacs2.3. The Col-0 plants were inoculated using droplets with $B$. cinerea mixed with the microbes undiluted (undil) or diluted to $1 / 10$ and $1 / 20$ in PDB $1 / 4(n=48 ; \pm$ SE). The average lesion size was determined $3 \mathrm{~d}$ after inoculation with $B$. cinerea. Different letters above each bar represent statistically significant differences (Tukey's test; $P<0.05)$. The experiment was repeated twice with similar results.

(referred to as strains 1, 2 and 3) and tested them individually on WT Col-0 plants against $B$. cinerea. When applied to nonsterile leaf surfaces of WT Col-0 plants, the bacterial strains 1 and 2 gave a partial (c. 33\%) protection, whereas strain 3 gave c. $84 \%$ protection to B. cinerea (Fig. 6). When plated on King's B medium, strain 3 displayed a fluorescent halo when viewed under UV light, so we presumed that it belongs to the genus Pseudomonas. The hyper variable region $16 \mathrm{~S}$ of these three strains was sequenced: strains 1 and 3 correspond to the genus Pseudomonas and strain 2 could be assigned to the genus Bacillus. Strains 1 and 3 were studied further and sequenced on the Pacific

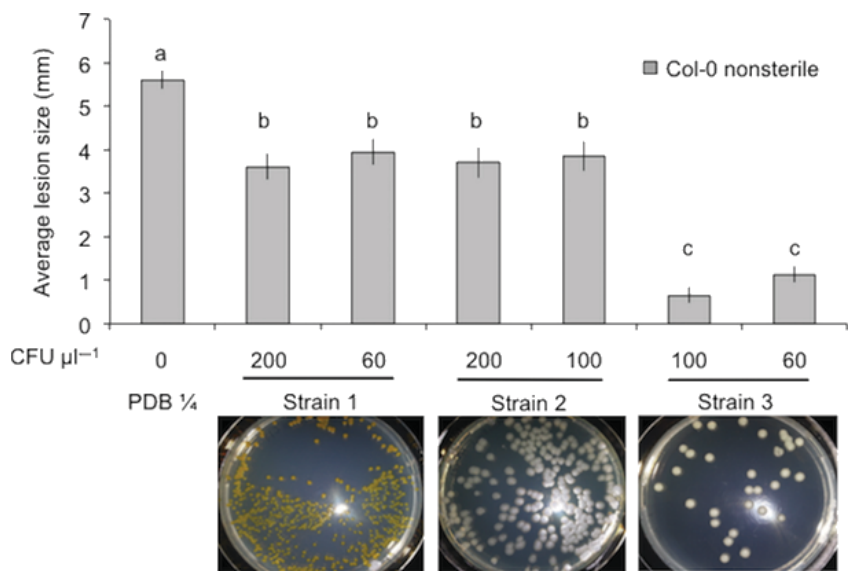

Fig. 6 Effect of individual microbial strains extracted from the phyllosphere of $b d g$ mutant on the resistance of Arabidopsis thaliana Col-0 plants to Botrytis cinerea. The bacterial strains 1, 2 or 3 isolated from surface washes of $b d g$ mutants were diluted in PDB $1 / 4$ at different concentrations (in colony-forming units (CFU) $\mu \mathrm{I}^{-1}$ ) then mixed with $B$. cinerea and inoculated with droplets on Col-0 plants ( $n=24 ; \pm$ SE). The average lesion size was determined $3 \mathrm{~d}$ after inoculation with $B$. cinerea. A representative picture of the bacterial strains is placed below each histogram. Different letters above each bar represent statistically significant differences (Tukey's test; $P<0.05)$. The experiment was carried out three times with similar results.
Biosciences sequencer of the Lausanne Genomic Technologies Facility. The library was prepared according to the manufacturer's recommendations and sequenced on a single SMRT cell. The coverage of the raw data is $c .150 \times$. We assembled the reads using the SMRT Pipeline with the HGAP3 protocol and obtained for strain 3 a single contig of 6374437 bp with a GC content of $59.23 \%$. No plasmid was detected. With reference to public databases, we found P.sp. URMO17WK12:I12 (Genbank AC: GCA_000514395.1) to be a very close draft genome of 33 contigs with a size of $6568431 \mathrm{bp}$ and GC content of $59.1 \%$ (ANI vs strain $3=99.56 \%$ ). The closest finished genome is P. mandeliistrain JR-1 (genome sequence of cold-adapted P. mandelii strain JR-1 (Jang et al., 2012)), but it is more distantly related $(\mathrm{ANI}=87.9 \%)$ as shown in Fig. S6(a,b). Strain 1 was assembled similarly to a single contig of 4835345 bp with a GC content of $61.7 \%$. No plasmid was detected. In the public databases, we found P. sp. URMO17WK12:I11 (Genbank acc. no. GCA_000514235.1), a very close draft genome of 44 contigs with a size of $4693207 \mathrm{bp}$ and a GC content of $61.9 \%$ (ANI vs strain $1=99.59 \%$ ) as shown in Fig. S6(c). We therefore named strain 3 as P. sp. friburgensis. P. sp. friburgensis was active when inoculated on WT plants, showing that its effect is dose dependent and can take place alone without other members of the bacterial community (Fig. S7). P. sp. friburgensis could act independently of other members of the community because it was also active on WT plants and $b d g$ mutants grown under sterile conditions (Fig. S8). Interestingly, PCR analysis using primers specific for P.sp. friburgensis showed that this strain was most abundant in $b d g$ mutant plants whereas strain 3 is present at much lower amounts in WT Col-0 and lacs2.3 (Fig. 7). This result underlines once more the qualitative differences in the composition of the bacterial population of the phyllosphere. The result in Fig. 3 shows that the Pseudomonas genus as a whole is more abundant in both cuticle mutants. Because neither strains 1, 2 nor P.sp. friburgensis showed a direct activity against $B$. cinerea, we tested if $P$. sp. friburgensis still could protect after boiling. Our results showed that after boiling the biological activity was lost (Fig. S9). The same results were observed with boiled phyllosphere microbes extracted from Col-0, bdg and lacs 2.3 (data not shown). Therefore, the effect of P. sp. friburgensis on

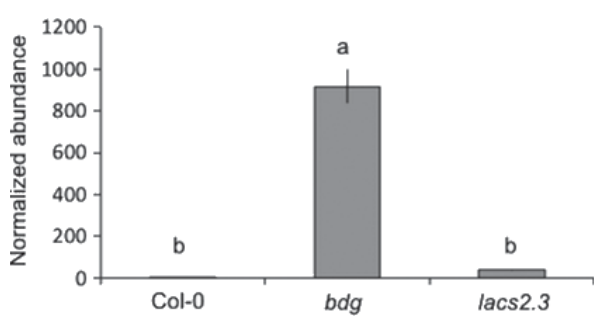

Fig. 7 Relative quantification of DNA from strain 3 (Pseudomonas sp. friburgensis) in Arabidopsis thaliana Col-0 plants and cuticle mutants. DNA from Col-0, bdg, lacs2.3 leaves were extracted and the relative quantification of strain 3 DNA in the different samples were determined by qPCR $(n=3 ; \pm S D)$. Different letters above each bar represent statistically significant differences (Tukey's test; $P<0.05$ ). The experiment was carried out three times with similar results. 
the resistance of the plant is heat-labile. In addition, we tested a filtered $(0.2 \mu \mathrm{m})$ culture of $P$. sp. friburgensis in PDB $1 / 4$ incubated $3 \mathrm{~d}$ in growth chamber (corresponding to the infection timing). In this condition, no active molecules induced the resistance of the plant against $B$. cinerea (data not shown). The effect of $P$. sp. friburgensis was still effective in mutants affected in salicylic acid (SA), jasmonic acid (JA) and ethylene (ET) signalling (Fig. S10) and in mutants of receptors for PAMPS such as fls2 and efr compared to WT Col-0 (Fig. S11).

\section{Discussion}

The $b d g$ and lacs2.3 mutants of Arabidopsis thaliana exhibit alterations in the architecture of the cuticle that typically lead to increased permeability and resistance to Botrytis cinerea (Bessire et al., 2007; Serrano etal., 2014). These mutants provided an interesting starting point for the work described here, and the question was asked whether the increased resistance in cuticle mutants could be explained by changes in the microbiome of the phyllosphere. To test this, we determined the resistance of plants under sterile conditions. Under these conditions, the resistance exhibited by the $b d g$ mutant was lost to a large extent, whereas lacs 2.3 retained full resistance (Fig. 1). The conditions used for sterile conditions are different than those in nonsterile pots, but the plants can nevertheless be inoculated and show typical grey mould symptoms (Fig. 1). The lesion size correlated with the growth of $B$. cinerea in Col- 0 and $b d g$ grown in sterile conditions (Fig. S1). Microbes of the phyllosphere were washed off leaves of unsterile plants and applied to leaves of the same plant type grown under sterile conditions before inoculation. The $b d g$ mutant pretreated with microbes regained resistance to $B$. cinerea at a comparable level as that observed under nonsterile conditions, demonstrating the importance of microbes of the phyllosphere for resistance (Fig. 2). The resistance of lacs 2.3 is likely to be controlled by endogenous factors because it remained resistant under sterile conditions although an additional effect by phyllosphere microbes cannot be completely ruled out (Figs 1,2).

This observation led us to explore the microbial diversity of the phyllosphere from these plants and possibly determine if the resistance could be related to any particular resident species. Sequencing of $16 \mathrm{~S}$ rDNA amplicons indicated that the most abundant bacterial taxa in wild-type (WT) Col-0 plants and in cuticle mutants belonged to the Proteobacteria phylum (Burkholderia, Pseudomonas, Rhizobium and Methylophilus) (Figs 3, S3). The relative proportion of these four genera was clearly different between the potting soil and in the plant phyllosphere. Most likely, the microbes are recruited from the soil, but other sources (air, handling) cannot be excluded. Overall, a relative consistency was observed among the genera on the different plants (Figs 3, 4, S3). Burkholderia, Pseudomonas and Methylophilus are among the major genera reported in the phylloplane of $A$. thaliana and other plants (Vorholt, 2012; Reisberg et al., 2013; Bodenhausen et al., 2014). Obviously, the physical and biochemical properties of the plant leaf surface, as well as environmental factors determine the composition of the microbes it harbours (Vorholt, 2012; Copeland et al., 2015).
The relative abundance of the four genera in the WT Col-0 was distinct from the cuticle mutants (Figs 3, S3). Because common features characterize both mutants but above all an increased permeability, this feature might possibly affect the hydration, the nutritional properties of the surface or the surface architecture, providing a specific niche for microbes. A dendrogram analysis that takes into account all samples (e.g. five samples per plant type and three replicates) leads to similar conclusions (Fig. 4). The bacterial community present on the washed surfaces of WT Col-0 can be distinguished from those on the cuticular mutants, and the bacterial community of $b d g$ and lacs 2.3 are clustering closely together without a specific pattern (Fig. 4).

Can bacteria from the phyllosphere of the $b d g$ mutant also protect WT plants grown under nonsterile conditions? This question assumes that the bacterial community of the $b d g$ mutant contains one or several unique species that might have the power to interfere with the interaction between $A$. thaliana and $B$. cinerea. Bacteria were washed off a determined surface area from a source plant and applied to a similar surface on the leaf of a recipient plant (see Materials and Methods) allowing for comparisons between leaf washes from different plant types. The undiluted leaf washes originating from $b d g$ or lacs 2.3 had a strong protective effect against $B$. cinerea, an effect that diminished upon dilution more so in washes from $b d g$ than from lacs2.3 (Fig. 5). This observation might be explained by the specific composition of the bacterial community on leaves of cuticular mutants and implies the occurrence of species with protective properties in the phyllosphere of cuticular mutants. The resistance observed is not due to a direct activity of the bacterial community on the spore germination (Fig. S5). Besides, a simple increase in the amount bacteria of the phyllosphere could also be involved in the protection, because WT Col-0 plants treated with a supplement of WT Col-0 bacteria also resulted in a minor increase in resistance to B. cinerea (Fig. 5). Overall, the colony-forming units ( $\mathrm{CFU} \mathrm{cm} \mathrm{cm}^{-2}$ ) were indeed higher in lacs 2.3 compared to WT Col-0, whereas the CFU $\mathrm{cm}^{-2}$ of $b d g$ was intermediate between lacs2.3 and Col-0 (Fig. S12).

We isolated 30 colonies from the phyllosphere of $b d g$ based on their morphology and colour. Of those we picked out three isolates that appeared conspicuous (yellow colour for isolate 1, matt surface for isolate 2 and shiny surface for isolate 3; see Fig. 6), carried out protection assays and found one strain to be active in protecting A.thaliana WT Col-0 against B. cinerea whether applied to sterile or nonsterile leaf surfaces (Figs 6, S7, S8). The $16 \mathrm{~S}$ hyper variable region of these three isolates corresponds to the genus Pseudomonas for the strains 1 and 3 and to the genus Bacillus for strain 2. The genus Pseudomonas is present among the genera isolated from $b d g$ phyllosphere, whereas the genus Bacillus was not detected (Figs 3, 4, S3). This observation suggests that strain 2 might be present in very low abundance, thus escaping detection. Alternatively, it might be a contamination. Thus, we were able to isolate a species, strain 3, from the leaf washes of the $b d g$ phyllosphere that had the power to protect A. thaliana WT Col-0 against B. cinerea whether applied to sterile or nonsterile leaf surfaces (Figs 6, S7, S8). We referred to 
strain 3 as $P$. sp. friburgensis and this strain shows near identity $($ ANI $=99.56 \%)$ with an unpublished draft genome for $P$. sp. URMO17WK12:I12 (Fig. S6). Interestingly this bacterium was also isolated from corn (R. Ley, pers. comm.), but its possible biological function has not been studied. The action of $P$. sp. friburgensis is indirect because it has no toxic effect when co-incubated with $B$. cinerea in vitro (data not shown). Antibiosis might, however, still take place on the phyllosphere where nutritional conditions are different. Also, other effects such as competition for iron or other ions or molecules might also take place. The production or release of a putative active compound requires living cells, because treatments with boiled P.sp. friburgensis (Fig. S9) or phyllosphere microbes from Col-0, bdg and lacs2.3 (data not shown) completely abolished biological activity. However, a degradation of the compound by heat cannot be excluded. The loss of activity of a culture filtrate (filtered through a $0.2-\mu \mathrm{m}$ pore size) of P.sp. friburgensis indicates that such a compound is likely to be released in planta only (data not shown) or that living cells are required to induce the activity. The effect of $P$. sp. friburgensis was still effective in mutants affected in salicylic acid, jasmonic acid and ethylene signalling compared to WT Col-0 (Fig. S10). An effect on the induction of plant resistance mechanisms would have to be sought elsewhere. The response to common bacterial pathogen-associated molecular patterns (PAMPs) such as flg22 and elf18 was also explored. Mutants of the receptors for flg22 or elf18 were still responsive to P. sp. friburgensis, indicating that this bacterium is detected by other receptors (Fig. S11). These intriguing observations need now to be followed up to understand how P. sp. friburgensis affects the defences of the plant. Importantly, the activity of P. sp. friburgensis also extended to other plant species, as shown by our experiments with apple (Fig. S13). Other observations have also documented the action of bacteria from the phyllosphere against foliar pathogens (Innerebner et al., 2011). Specific strains of P.cepacia have been shown to control B. cinerea and other postharvest diseases, but their mode of action has not always been well documented (Janisiewicz \& Korsten, 2002). A number of $P$. syringae strains isolated from the rhizosphere were shown to have an antagonistic activity against B. cinerea (Hernandez-Leon et al., 2015). They were found to produce antibiotics, some of which are volatile, as well as plant growthpromoting substances. Thus, the genus Pseudomonas appears to harbour interesting potential biocontrol strains with a relatively nonspecific host range that display a wide range of mechanisms of action (Santoyo et al., 2012).

The phyllosphere might be viewed as an external shell providing a first line of defence against mostly nonpathogens, whereas true pathogens have co-evolved to cope with it. Because the microbiomes are different from one plant species to another, changing the original composition of the microbes, for example with phyllosphere microbes from another species (crossinoculation) would be highly instructional to determine the importance of the microbiome of a plant species as a protective layer. These considerations made us aware that the microbiome of the outer boundary of plants has not really been taken into consideration in our studies on plant disease resistance mechanisms. Future work should be directed at exploring this promising area. To a certain degree our results are in tune with recent data on the function of microbiomes associated with other animals or humans. These data highlight the importance of the phyllosphere as a source of potential biological plant protectants. Efforts in searching for novel active strains against foliar pathogens should definitely target this outer layer of the leaves.

\section{Acknowledgements}

We thank Linda Grainger for her invaluable technical assistance, Laurent Poirel for useful discussions and Michael Stumpe for the statistics. This work was made possible by funds to J-P.M. from the Swiss National Science Foundation. Unyarat Ritpitakphong was supported by a fellowship of the Swiss Confederation, and Artit Vimoltust by a fellowship of Product \& Technology Development Center, SCG Paper plc. Thanks are also extended to Microsynth AG for the library preparation and Illumina sequencing; The Genomic Technologies Facility (GTF), Center for Integrative Genomics, University of Lausanne for the PacBio sequencing. The computations were performed at the Vital-IT (http://www.vital-it.ch) Center for high-performance computing of the SIB Swiss Institute of Bioinformatics.

\section{Author contributions}

J-P.M. and F.L. planned and designed the research. U.R., A.V., A.B., L.F. and F.L. performed experiments, conducted fieldwork and analysed data. F.L., L.F., U.R, A.V. and J-P.M. interpreted the data. J-P.M., F.L. and L.F. wrote the manuscript.

\section{References}

Alikhan N-F, Petty NK, Ben Zakour NL, Beatson SA. 2011. BLAST Ring Image Generator (BRIG): simple prokaryote genome comparisons. BMC Genomics 12: 402-412.

Andrews JH, Harris RF. 2000. The ecology and biogeography of microorganisms on plant surfaces. Annual Review of Phytopathology 38: 145-180.

Arndt D, Xia J, Liu Y, Zhou Y, Guo AC, Cruz JA, Sinelnikov I, Budwill K, Nesbo CL, Wishart DS. 2012. METAGENassist: a comprehensive web server for comparative metagenomics. Nucleic Acids Research 40: W88-W95.

Bessire M, Chassot C, Jacquat AC, Humphry M, Borel S, Petetot JMC, Metraux JP, Nawrath C. 2007. A permeable cuticle in Arabidopsis leads to a strong resistance to Botrytis cinerea. EMBO Journal 26: 2158-2168.

Biedermann L, Rogler G. 2015. The intestinal microbiota: its role in health and disease. European Journal of Pediatrics 174: 151-167.

Bodenhausen N, Bortfeld-Miller M, Ackermann M, Vorholt JA. 2014. A synthetic community approach reveals plant genotypes affecting the phyllosphere microbiota. PLoS Genetics 10: e1004283.

Bodenhausen N, Horton MW, Bergelson J. 2013. Bacterial communities associated with the leaves and the roots of Arabidopsis thaliana. PLoS ONE 8: e56329.

Bulgarelli D, Schlaeppi K, Spaepen S, van Themaat EVL, Schulze-Lefert P. 2013. Structure and functions of the bacterial microbiota of plants. Annual Review of Plant Biology 64: 807-838.

Chassot C, Nawrath C, Métraux J-P. 2007. Cuticular defects lead to full immunity to a major plant pathogen. Plant Journal 49: 972-980.

Chin C-S, Alexander DH, Marks P, Klammer AA, Drake J, Heiner C, Clum A, Copeland A, Huddleston J, Eichler EE et al. 2013. Nonhybrid, finished microbial genome assemblies from long-read SMRT sequencing data. Nature Methods 10: 563-569. 
Copeland JK, Yuan LJ, Layeghifard M, Wang PW, Guttman DS. 2015. Seasonal community succession of the phyllosphere microbiome. Molecular Plant-Microbe Interactions 28: 274-285.

Darling AE, Mau B, Perna NT. 2010. progressiveMauve: multiple genome alignment with gene gain, loss and rearrangement. PLOS ONE 5: e11147.

Dash S, Clarke G, Berk M, Jacka FN. 2015. The gut microbiome and diet in psychiatry: focus on depression. Current Opinion in Psychiatry 28: 1-6.

Engel P, Moran NA. 2013. Functional and evolutionary insights into the simple yet specific gut microbiota of the honey bee from metagenomic analysis. Gut Microbes 4: 60-65.

Foster JA, McVey Neufeld K-A. 2013. Gut-brain axis: how the microbiome influences anxiety and depression. Trends in Neurosciences 36: 305-312.

Gachon C, Saindrenan P. 2004. Real-time PCR monitoring of fungal development in Arabidopsis thaliana infected by Alternaria brassicicola and Botrytis cinerea. Plant Physiology and Biochemistry 42: 367-371.

Hernandez-Leon R, Rojas-Solis D, Contreras-Perez M, Orozco-Mosqueda MD, Macias-Rodriguez LI, Reyes-de la Cruz H, Valencia-Cantero E, Santoyo G. 2015. Characterization of the antifungal and plant growth-promoting effects of diffusible and volatile organic compounds produced by Pseudomonas fluorescens strains. Biological Control 81: 83-92.

Humphrey PT, Nguyen TT, Villalobos MM, Whiteman NK. 2014. Diversity and abundance of phyllosphere bacteria are linked to insect herbivory. Molecular Ecology 23: 1497-1515.

Huson DH, Mitra S, Ruscheweyh H-J, Weber N, Schuster SC. 2011. Integrative Integrative analysis of environmental sequences using MEGAN4. Genome Research 21: 1552-1560.

Innerebner G, Knief C, Vorholt JA. 2011. Protection of Arabidopsis thaliana against leaf-pathogenic Pseudomonas syringae by Sphingomonas strains in a controlled model system. Applied and Environmental Microbiology 77: 32023210.

Jang S-H, Kim J, Kim J, Hong S, Lee C. 2012. Genome sequence of coldadapted Pseudomonas mandelii strain JR-1. Journal of Bacteriology 194: 3263 3263.

Janisiewicz WJ, Korsten L. 2002. Biological control of postharvest diseases of fruits. Annual Review of Phytopathology 40: 411-441.

Kostic AD, Howitt MR, Garrett WS. 2013. Exploring host-microbiota interactions in animal models and humans. Genes \& Development 27: 701-718.

Kurdyukov S, Faust A, Nawrath C, Bär S, Voisin D, Efremova N, Franke R, Schreiber L, Saedler H, Metraux J-P et al. 2006. The Epidermis-specific extracellular BODYGUARD controls cuticle development and morphogenesis in Arabidopsis. Plant Cell 18: 321-339.

Lindow SE, Brandl MT. 2003. Microbiology of the phyllosphere. Applied Environmental Microbiology 69: 1875-1883.

von Malek B, van der Graaff E, Schneitz K, Keller B. 2002. The Arabidopsis male-sterile mutant dde2-2 is defective in the ALLENE OXIDE SYNTHASE gene encoding one of the key enzymes of the jasmonic acid biosynthesis pathway. Planta 216: 187-192.

Quast C, Pruesse E, Yilmaz P, Gerken J, Schweer T, Yarza P, Peplies J, Gloeckner FO. 2013. The SILVA ribosomal RNA gene database project: improved data processing and web-based tools. Nucleic Acids Research 41: D590-D596.

Reisberg EE, Hildebrandt U, Riederer M, Hentschel U. 2013. Distinct phyllosphere bacterial communities on Arabidopsis wax mutant leaves. PLoS ONE 8: e78613.

Sabree ZL, Moran NA. 2014. Host-specific assemblages typify gut microbial communities of related insect species. SpringerPlus 3: 138-138.

Santoyo G, Orozco-Mosqueda MdC, Govindappa M. 2012. Mechanisms of biocontrol and plant growth-promoting activity in soil bacterial species of Bacillus and Pseudomonas: a review. Biocontrol Science and Technology 22: 855872.

Schloss PD, Westcott SL, Ryabin T, Hall JR, Hartmann M, Hollister EB, Lesniewski RA, Oakley BB, Parks DH, Robinson CJ et al. 2009. Introducing mothur: open-source, platform-independent, community-supported software for describing and comparing microbial communities. Applied and Environmental Microbiology 75: 7537-7541.

Seemann T. 2014. Prokka: rapid prokaryotic genome annotation. Bioinformatics 30: $2068-2069$
Serrano M, Torres M, Coluccia F, L'Haridon F, Metraux J-P. 2014. The cuticle and plant defense to pathogens. Frontiers in Plant Science 5: 4-8.

Silvester N, Alako B, Amid C, Cerdeño-Tárraga A, Cleland I, Gibson R, Goodgame N, ten Hoopen P, Kay S, Leinonen R et al. 2014. Content discovery and retrieval services at the European Nucleotide Archive. Nucleic Acids Research 43: D23-D29.

Viaud S, Daillere R, Boneca IG, Lepage P, Langella P, Chamaillard M, Pittet MJ, Ghiringhelli F, Trinchieri G, Goldszmid R et al. 2015. Gut microbiome and anticancer immune response: really hot $\mathrm{Sh}(\mathrm{star}) \mathrm{t} !$. Cell Death and Differentiation 22: 199-214.

Vorholt JA. 2012. Microbial life in the phyllosphere. Nature Review Microbiology 10: 828-840.

Walker TS, Bais HP, Grotewold E, Vivanco JM. 2003. Root exudation and rhizosphere biology. Plant Physiology 132: 44-51.

Wang Q, Garrity GM, Tiedje JM, Cole JR. 2007. Naive Bayesian classifier for rapid assignment of rRNA sequences into the new bacterial taxonomy. Applied and Environmental Microbiology 73: 5261-5267.

White JR, Nagarajan N, Pop M. 2009. Statistical methods for detecting differentially abundant features in clinical metagenomic samples. PLoS Computational Biology 5: e1000352.

Zipfel C, Kunze G, Chinchilla D, Caniard A, Jones JDG, Boller T, Felix G. 2006. Perception of the bacterial PAMP EF-Tu by the receptor EFR restricts Agrobacterium-mediated transformation. Cell 125: 749-760.

Zipfel C, Robatzek S, Navarro L, Oakeley EJ, Jones JDG, Felix G, Boller T. 2004. Bacterial disease resistance in Arabidopsis through flagellin perception. Nature 428: 764-767.

\section{Supporting Information}

Additional supporting information may be found in the online version of this article.

Fig. S1 The growth of B. cinerea on leaves of A. thaliana Col-0, $b d g$ and lacs 2.3 in sterile and nonsterile conditions determined by trypan blue staining.

Fig. S2 Rarefaction curves.

Fig. S3 Top 20 most abundant genera in $b d g$ and the relative abundance in $A$. thaliana Col-0 plants, lacs2-3 and soil samples compared to $b d g$.

Fig. S4 Effect of surface washes of leaves from sterile $A$. thaliana Col-0 plants and cuticle mutants on the resistance of nonsterile Col-0 plants to B. cinerea.

Fig. S5 The activity of phyllosphere microbes from $A$. thaliana Col- $0, b d g$ and lacs 2.3 on the spore germination of $B$. cinerea in vitro.

Fig. S6 Genome of strain 3 and strain 1.

Fig. S7 Dose-dependent effect of strain 3 (P. sp. friburgensis) on the resistance of $A$. thaliana Col-0 plants to $B$. cinerea.

Fig. S8 Effect of strain 3 (P. sp. friburgensis) on the resistance of sterile $A$. thaliana Col-0 plants and cuticle mutants to $B$. cinerea.

Fig. S9 Effect of boiled strain 3 (P. sp. friburgensis) on the resistance of $A$. thaliana Col-0 plants to $B$. cinerea. 
Fig. S10 Effect of strain 3 (P. sp. friburgensis) on the resistance of A. thaliana Col-0 plants and SA-, JA- and ET- signalling mutants to $B$. cinerea.

Fig. S11 Effect of strain 3 (P.sp. friburgensis) on the resistance of A. thaliana Col-0 plants and PAMP receptor mutants to B. cinerea.

Fig. S12 Average of colony forming units (CFU) $\mathrm{cm}^{-2}$ extracted from the phyllosphere of A. thaliana Col-0 plants and cuticle mutants.
Fig. S13 Effect of strain 3 (P. sp. friburgensis) on the resistance of apple fruit to $B$. cinerea. 